\title{
INFORMATIZAÇÃO DE COLEÇÕES UTILIZANDO UM SISTEMA DE INFORMAÇÕES GEOGRÁFICAS (SIG): UM PROTÓTIPO DESENVOLVIDO PARA A COLEÇÃO DE LEPIDOPTERA DO DEPARTAMENTO DE ZOOLOGIA DA UNIVERSIDADE FEDERAL DO PARANÁ 1
}

\author{
Renato Roxo Coutinho Dutra ${ }^{2}$ \\ Marcelo Antunes Araújo ${ }^{3}$
}

\begin{abstract}
THE INFORMATIZATION OF COLLECTIONS USING A GEOGRAPHIC INFORMATION SYSTEM (GIS): A PROTOTYPE DEVELOPED FOR THE BUTTERFLIES' COLLECTION OF THE "Departamento de Zoologia, Universidade Federal do Parana". The purpose of this paper is to present a prototype, which was developed using an ARC/INFO software, for the informatization of Butterflies' Collection of the "Departamento de Zoologia da Universidade Federal do Paraná".

KEY WORDS. Collection, informatization, Geographic Information System, GIS
\end{abstract}

\section{O conceito tradicional de Museu}

Segundo QueIroz (1987): "a imagem do museu como um local silencioso e empoierado, em que se dispõe, de maneira mais ou menos ordenada, objetos e documentos de passadas eras, é a mais corrente; a esta noção de "depósito" se adiciona uma outra, a de exposição didática, mas nestas duas finalidades parecem se esgotar as idéias a respeito destas entidades. A maioria dos museus do país foi formada sob essa dupla visão, que é ainda dominante na mentalidade de grande parte dos administradores e políticos nacionais."

\section{Uma nova visão sobre o papel dos Museus}

Para THOMÉ (1988): "o conhecimento do patrimônio natural, através do seu inventário criterioso e sistematizado, bem como a avaliação para detectar seu manejo, deve ser altamente prioritário e nos parece ser uma das finalidades mais preciosas de um Museu de História Natural contemporâneo (...) A seguir deve-se conseguir convencer as autoridades constituídas e bem assim a população como um todo, de que a atividade museológica como banco de dados não é só necessária, mas altamente prioritária, indispensável para se chegar ao amanhã dentro de um contexto são e auto-sustentável para a sobrevivência da humanidade."

1) Contribuição número 960 do Departamento de Zoologia, Universidade Federal do Paraná.

2) Escola Técnica, Universidade Federal do Paraná. Rua Alcides Vieira Arcoverde 1225, 81520-260 Curitiba, Paraná, Brasil.

3) Jari Celulose S.A.. 68240-000 Monte Dourado, Pará, Brasil. 
"Este acúmulo de dados, organizado e dinamizado, torna os museus ferramenta prioritária no planejamento integrado do desenvolvimento, tanto regional, como universal.” (THOMÉ 1985)

\section{A importância das Coleções existentes nas Universidades}

Para MARTINS (1988): “as instituições que conservam coleções zoológicas, produzem pesquisa científica com fundamento nesse acêrvo, formam e/ou preparam pessoal especializado e veiculam cultura à população, são das mais variadas naturezas, nem sempre um Museu, embora esses sejam os atributos de um museu moderno (...). Entretanto, no nosso país e certamente em muitos outros, a maioria dos núcleos de pesquisa zoológica nas universidades está estabelecida em Departamentos, cujos acêrvos não podem ser rotulados como "Museus"."

Segundo MARINONI et al. (1992): “o Departamento de Zoologia da Universidade Federal do Paraná, desde o seus primórdios, teve uma linha de pesquisa fundamentada em Sistemática de Insetos, através do trabalho do seu Professor Catedrático, Padre Jesus Santiago Moure.

Foi a partir de 1956 que a coleção entomológica passou a ser formada, pois, até então, o material coletado era depositado junto à coleção do antigo Museu Paranaense (...). Hoje, a Coleção de Entomologia Pe. Jesus S.Moure do Departamento de Zoologia da Universidade Federal (DZUP), conta com mais de 5.000 .000 de exemplares..."

Segundo MARTINS (in PAPAVERo 1983): “a coleção taxonômica é a reunião ordenada de espécimes mortos ou partes corporais desses espécimes, devidamente preservados para estudos (...) A parte da Biologia que visa a classificação dos animais, Taxonomia, fundamenta-se principalmente na análise comparativa de seus atributos (caracteres taxonômicos) (...) Caracteres morfológicos frequentemente podem ser analisados em espécimes preservados e, com raríssimas exceções, toda a classificação animal se fundamenta no estudo comparativo desses caracteres. Fica claro então que a coleção é imprescindível para estudos taxonômicos."

Ao descrever uma nova espécie, o zoólogo deverá designar claramente um único espécime como "holótipo", o qual representa o padrão de referência que determina a aplicação de um nome científico, servindo não apenas para possibilitar a identificação e reconhecimento da nova entidade, como para tornar esse novo nome válido (BERNARDI in PAPAVERo 1983).

Ainda, o zoólogo que designa um tipo deve depositá-lo num museu ou outra instituição pública em que seja preservado com segurança e acessível para fins de pesquisa, sendo que toda instituição em que há tipos depositados deve, entre outras de suas responsabilidades: 1) publicar listas de material-tipo em sua posse e sob sua custódia e; 2) na medida do possível, comunicar informações sobre os tipos aos zoólogos que as requisitem (BERNARDi in PAPAVERO 1983).

Como informatizar as Coleções do Departamento de Zoologia da Universidade Federal do Paraná, tornando-as mais acessíveis à comunidade científica e à população

Apesar de não estarem abertas à visitação pública, por se tratarem de coleções científicas que abrigam no seu acervo um grande número de material-tipo, de grande 
valor científico, as coleções de insetos existentes no Departamento de Zoologia da Universidade Federal do Paraná trazem no seu âmago um valor cultural inestimável, obrigando procurar e propor soluções que tornem viável o seu acesso à população.

Por outro lado, vê-se que são atributos das instituições em que há tipos depositados, a publicação de listas de material-tipo em sua posse e, na medida do possível, a comunicação destas informações quando solicitadas.

Se até o momento não houve uma preocupação por parte do Departamento de Zoologia da Universidade Federal do Paraná em propor soluções para tornar suas coleções acessíveis à população, de forma a divulgar as atividades de um museu, conforme propugnado no XV Congresso Brasileiro de Zoologia, propostas de informatização das mesmas foram apresentadas por CURE \& DUTRA (1990).

Um exemplo de publicação de lista de material-tipo de Coleoptera (besouros) pode ser encontrado em MARINONI et al. (1992).

Nos dois trabalhos acima citados, os autores preocuparam-se exclusivamente com as informações alfanuméricas ali armazenadas. Em nenhum momento aventou-se a possibilidade de se armazenar num Banco de Dados, também, a informação visual ali disponível que, se não é tão importante para alguns grupos de insetos, é de importância fundamental para os estudiosos de borboletas.

Assim, diante dos argumentos apresentados, e levando em consideração a plasticidade das borboletas, o que em muitos casos fez com que espécies de extrema beleza fôssem extintas ( $c f$. CASAGRANDE \& MIELKE 1992), sugere-se, de forma a tornar factível esta interação Universidade-População-Comunidade científica, a utilização de um Sistema de Informações Geográficas (SIG) para esta finalidade.

\section{MATERIAL E MÉTODOS}

\section{Base Gráfica}

O aplicativo foi desenvolvido nas dependências do Centro Integrado de Estudos em Geoprocessamento (CIEG) -Universidade Federal do Paraná. Utilizouse o programa ARC/INFO v.7.0.2. (Environmental Systems Research Institute ESRI), rodando em uma Workstation HP, com sistema operacional UNIX.

Foram utilizadas três bases de referência espacial, digitalizadas no AutoCAD r.12: 1) planta plani-altimétrica cadastral (escala 1:500, datada de junho/83) do Setor de Ciências Biológicas da Universidade Federal do Paraná, obtida junto à Prefeitura do Centro Politécnico; 2) plantas dos três pavimentos (escala 1:125, datadas de maio/93 -folha 04-16 e de junho/93 - folhas 05-16 e 06-16) do Departamento de Zoologia - Setor de Ciências Biológicas -UFPR, também obtidas junto à Prefeitura do Centro Politécnico e digitalizadas em perspectiva, o que permitiu a visualização dos três andares concomitantemente e, 3) armário Entomológico, digitalizado a partir de medições efetivadas pelos autores no interior da Coleção de Lepidoptera do Departamento de Zoologia da Universidade Federal do Paraná.

As imagens das borboletas (14 espécies em vista ventral e dorsal), associadas à última base mencionada, foram obtidas a partir da digitalização de fotografias; para tal, utilizamos um scanner de mesa (ScanJet II C - Hewlett Packard) e o 
programa Deskscan II; as 14 espécies utilizada neste trabalho foram: Agrias claudina Staudinger, 1855; Alesa prema (Godart, 1824); Arcas ducalis (Westwood, 1851); Callicore sorana (Godart, 1823); Dasyophthalma rusina Godart, [1824]; Elbella polyzona (Latreille, [1824]); Helicopis acis (Fabricius, 1782); Microceris variicolor (Ménétriés, 1855); Mimoniades montana Zikán, 1938; Nessaea hewitsoni Felder, 1859; Orobrassolis ornamentalis (Stichel, 1906); Prepona deiphile (Godart, 1824); Pseudocroniades machaon (Westwood, [1852]) e Symmachia arion (Felder, 1865).

\section{Base Alfanumérica}

Os dados alfanuméricos referentes às salas do Departamento de Zoologia (uso, usuário e número da sala) foram obtidos no próprio Departamento.

Os dados relativos às borboletas foram obtidos junto aos próprios Curadores da Coleção, considerando-se, principalmente, os seguintes aspectos: nome científico, família, subfamília, sexo, envergadura alar e sua localização na Coleção (armário, coluna e gaveta onde está depositada a espécie). Como fontes de referência, também utilizamos os trabalhos de CASAGRANDE \& MielKe (1992) e MielKe (1994), onde constam informações sobre nove das quatorze borboletas utilizadas no desenvolvimento deste protótipo.

\section{Criação da Topologia}

A transferência dos arquivos, em padrão.dxf, gerados a partir dos layers digitalizados no AutoCAD, para o padrão ARC/INFO, foi feita através do comando dxfarc. Durante esta transferência, alguns procedimentos importantes foram: anotar o nome do layer que originou o arquivo.dxf e se o mesmo era um arquivo de pontos ou de arcos.

Posteriormente, foi gerada uma coverage a partir de cada layer, utilizandose, conforme o caso, os comandos build ou clean.

\section{Tabelas}

Além das Tabelas geradas pelo próprio ARC/INFO (e.g.AAT,.PAT, entre outras), quando da criação da topologia, foram geradas, através do TABLES, mais duas Tabelas: 1) SALAS.DAT, com a seguinte estrutura: salas-id, 2, 3, B; uso, 16, 17, C; usuário, 30, 31, C, numero, 4, 5, C e; 2) GAVETAS.DAT, com a seguinte estrutura: gavetas-id, 4, 5, B; nome_cientifico, 42, 43, C; familia, 11, 12, C; subfamilia, 13, 14, C; sexo, 5, 6, C; envergadura_alar, 7, 8, C e, localizacao, 8, 9, C.

Os dados relativos a cada um destes campos, para as coverages correspondentes, foram digitados a partir do Editor de Textos disponível na HP, em arquivos ASCII com extensão.txt (e.g. salas.txt e gavetas.txt). Posteriormente, através do comando add from (e.g. sel gavetas.dat; add from gavetas.txt) essas informações foram implementadas nas Tabelas cuja estrutura havia sido definida anteriormente. Finalmente, através do comando joinitem (e.g. joinitem gavetas.pat gavetas.dat gavetas.pat gavetas-id gavetas-id), essas informações foram agregadas às Tabelas.PAT correspondentes. 
Este procedimento também foi utilizado para agregar a Tabela pontos.xcode (contendo o campo dxf-elevation) à Tabela pontos.pat, quando da criação do MDT (Modelo Digital do Terreno) para a área do Setor de Ciências Biológicas.

\section{Programação com AML (Arc Macro Language)}

O aplicativo, para o seu funcionamento, foi programado através da linguagem AML (Arc Macro Language) (ESRI 1994), visando a todo momento tornar a interface sistema/usuário o mais amigável possível. Através do editor de textos do próprio UNIX, foram criadas várias macros, que se concatenavam.

Da mesma forma, foram criados os vários menus utilizados no aplicativo.

\section{RESULTADOS E DISCUSSÃO}

\section{O aplicativo e seu funcionamento}

A figura 1 mostra algumas das imagens digitalizadas que constituem o Banco de Imagens deste aplicativo.

O aplicativo apresenta, inicialmente, uma coverage do Setor de Ciências Biológicas da Universidade Federal do Paraná (Fig. 2) e, na sequência, um Modelo Digital do Terreno (MDT) da área onde está localizado o Setor (Fig. 3); existe um menu suspenso que acompanha o MDT e que permite nos deslocarmos para o interior do Departamento de Zoologia, que no caso é o Departamento que nos interessa, ou para qualquer um dos Departamentos do Setor.

A coverage do Departamento de Zoologia (Fig. 4) permite consultas que podem ser feitas através do menu suspenso que a acompanha; entre as várias opções de consulta, pode-se verificar as informações sobre uma determinada sala, escolhida através do cursor, informações sobre as salas dos Professores Seniores, sobre a localização das salas de aula, por exemplo, ou sobre a Coleção de Lepidoptera (e.g. curadores, coleção, localização, etc.).

Ao desenharmos a coverage da Coleção de Lepidoptera (um dos armários entomológicos e suas gavetas; ao todo, seriam 19 armários, cada um com oito colunas e cada coluna com 33 gavetas), ativamos o menu que permite obter informações sobre uma determinada gaveta (Fig. 5), ou pesquisar sobre uma das borboletas cujas informações/localização, além das fotos ventral e dorsal (Fig. 6), foram implementadas. Cada escolha remeterá o usuário para um novo menu suspenso, em função da Família escolhida.

\section{Considerações finais}

Uma das várias definições de SIG é: Um conjunto sistematizado de hardware, software, dados geográficos e ante-projeto para que se possa, eficientemente, capturar, armazenar, atualizar, manipular, analisar e expor todas as formas de informação georreferenciada.

Assim, apesar de ter sido criado para atender às necessidades de áreas do conhecimento humano como cartografia, geografia, geologia ou planejamento urbano, por exemplo, o Programa ARC/INFO mostrou-se muito útil para gerenciar o acervo de coleções entomológicas, que eram objeto de nosso interesse. 

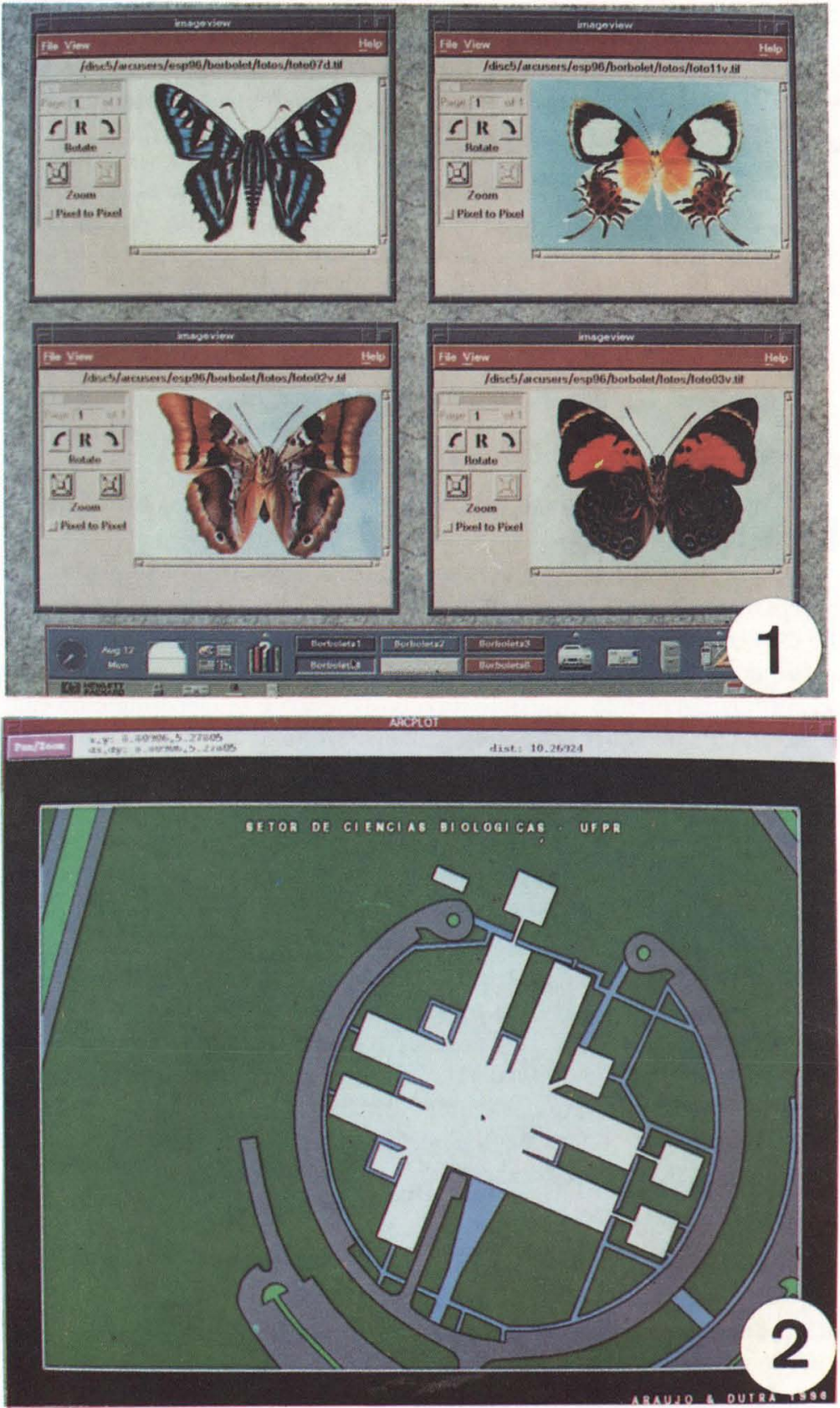

Figs 1-2. (1) Banco de Imagens digitalizadas, mostrando algumas fotografias que foram utilizadas no trabalho; (2) coverage do Setor de Ciências Biológicas da Universidade Federal do Paraná. 

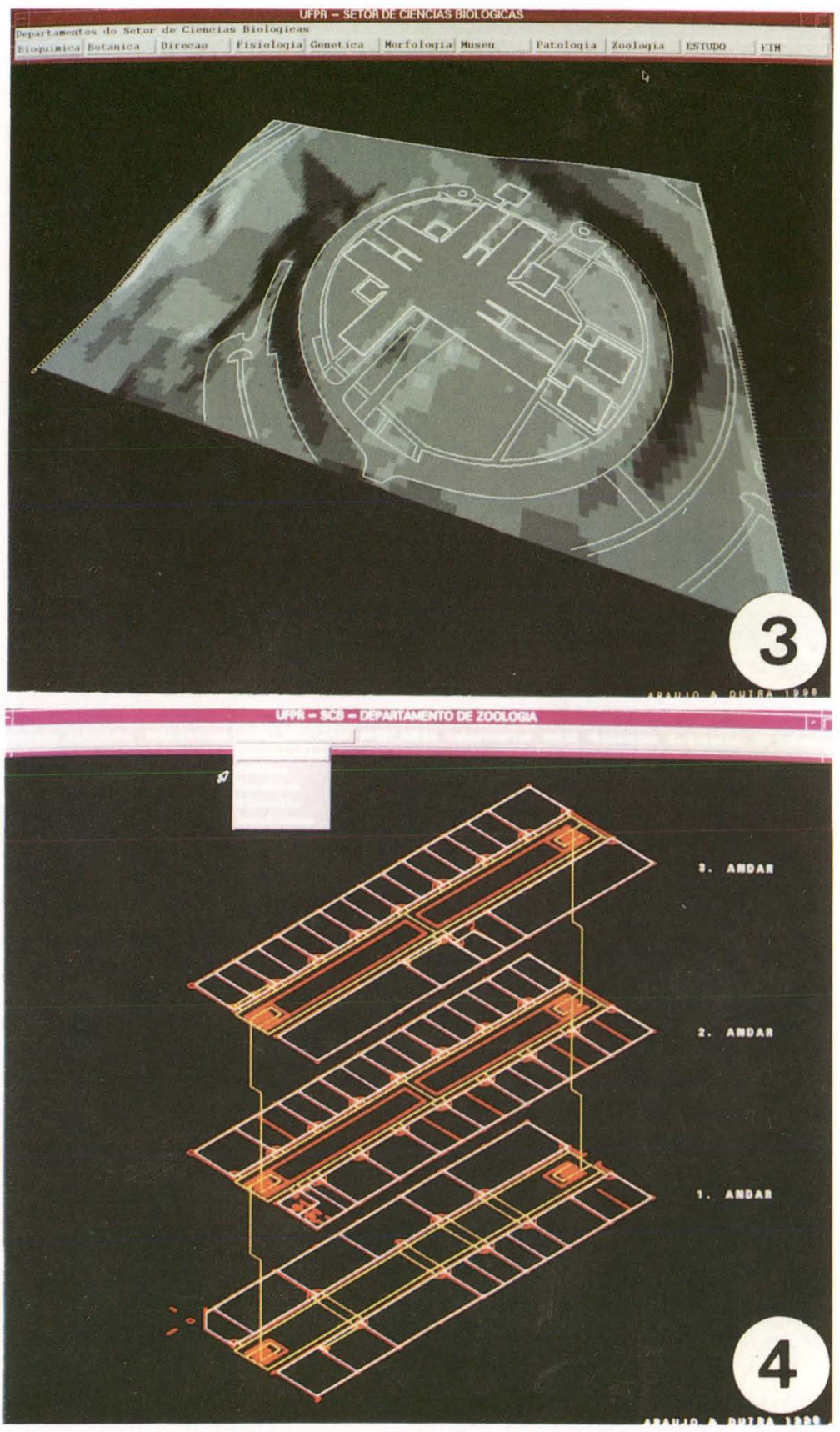

Figs 3-4. (4) Modelo Digital do Terreno (MDT) gerado para a área onde está localizado o Setor de Ciências Biológicas da Universidade Federal do Paraná; (4) coverage do Departamento de Zoologia da Universidade Federal do Paraná, selecionado através do menu suspenso. 

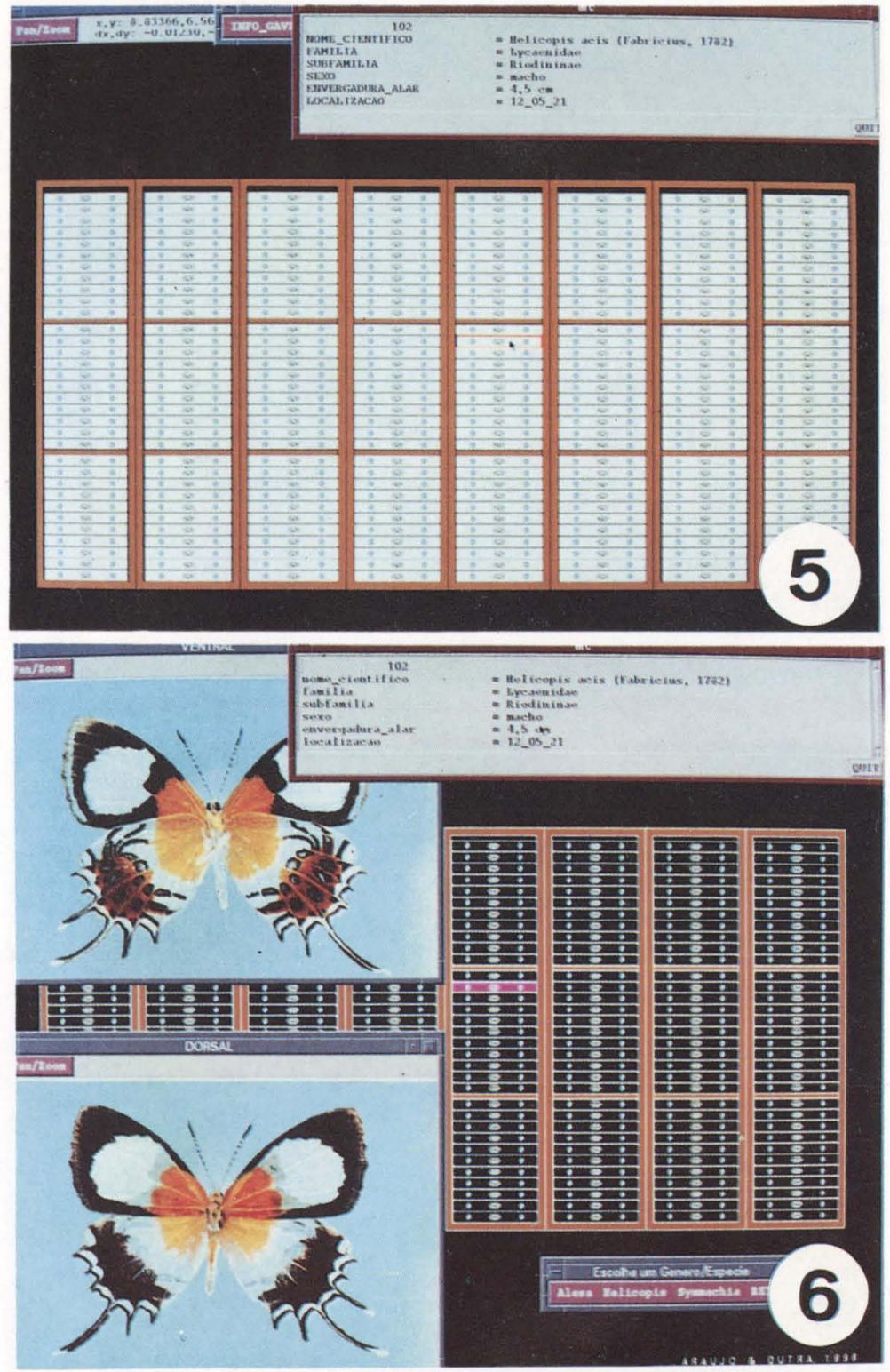

Figs 5-6. (5) Coverage de um dos armários entomológicos, e respectivas gavetas, da Coleção de Lepidoptera do Departamento de Zoologia da Universidade Federal do Paraná, destacando uma gaveta selecionada através do cursor, e mostrando as informações sobre a mesma; (6) vistas ventral e dorsal da espécie de borboleta selecionada através do menu, sua localização na Coleção e informações sobre a mesma. 
Segundo GoMEL \& CAMPOS (1995), "Manipular dados gráficos e alfa-numéricos, gerenciar grande quantidade de dados (como imagens digitalizadas), relacionando-os entre si e fornecer ao usuário uma facilidade de consulta, através de interfaces gráficas, são características de um Sistema de Informações Geográficas (SIG)." Ainda, segundo os autores, quando o Sistema de Informações Geográficas perde o referencial Geo e passa a ser um Sistema de Informações Gráficas ou Gráfico-espaciais, qualquer aplicação que necessite relacionar (com inteligência) informações gráficas e alfanuméricas, pode ser desenvolvida a partir de um SIG, de um passeio pelo acervo de um museu a um estudo do fluxo sanguíneo dentro do corpo humano.

$\mathrm{O}$ aprendizado do programa ARC/INFO demanda um tempo relativamente longo, e é de uso extremamente restrito, já que exige um equipamento com especificações que fogem ao comumente encontrado nas Universidades, além do seu custo também ser relativamente alto. Portanto, para o tipo de aplicação desenvolvida neste trabalho, sugerimos a utilização de outro software da Environmental Systems Research Institute (ESRI) para esta finalidade, o ArcView, que pode ser implementado em microcomputadores, de custo relativamente baixo e de fácil aprendizagem.

AGRADECIMENTOS. Aos Curadores da Coleção de Lepidoptera do Departamento de Zoologia da Universidade Federal do Paraná, Professores Dra Mirna M. Casagrande e Dr. Olaf H.H. Mielke, pela inestimável colaboração.

Dedicatória. Este trabalho é dedicado aos Professores Seniores do Departamento de Zoologia da Universidade Federal do Paraná.

\section{REFERÊNCIAS BIBLIOGRÁFICAS}

Casagrande, M.M. \& O.H.H. MielKe. 1992. Borboletas (Lepidoptera) ameaçadas de extinção no Paraná. Revta bras. Zool. 9 (1/2): 75-92.

CuRE, J.R. \& R.R.C. Dutra. 1990. Organização de Banco de Dados Zoológicos. Revta bras. Zool. 7 (4): 445-457.

ESRI. 1994. ARC Macro Language. Developing ARC/INFO menus and macros with AML ${ }^{\text {TM }}$. Redlands, California, Environmental Systems Research Institute Inc..

Gomel, D. \& R.F. Campos. 1995. Da Praça ao Museu. Revta Fator Gis 2 (8): 29-33.

Marinoni, R.C.; L.M. Almeida; D.S. NAPP \& G.H. Rosado-Neto. 1992. Primeira lista de material-tipo de Coleoptera da Coleção de Entomologia Pe. J.S. Moure, do Departamento de Zoologia da Universidade Federal do Paraná. Revta bras. Zool. 9 (1/2): 99-126.

MARTINS, U.R. 1988. Museus universitários. Revta bras. Zool. 5 (4): 623-627. MiELKE, O.H.H. 1994. Revisão de Elbella Evans e gêneros afins (Lepidoptera, Hesperiidae, Pyrrhopyginae. Revta bras. Zool. 11 (3): 395-586.

PAPAVERO, N. 1983. Fundamentos práticos de taxonomia zoológica: coleções, 
bibliografia, nomenclatura. Belém, Ed. Museu Paraense Emílio Goeldi, 252p. QueIroz, M.I.P. 1987. Museus no Brasil. Ciência e Cultura 39 (2): 115.

THOMÉ, J.W. 1985. Museus, sustentáculos do desenvolvimento. Ciência e Cultura 37 (1):61-63.

1988. Os museus estaduais. Revta bras. Zool. 5 (4): 629-631.

Recebido em 22.VIII.1996; aceito em 18.VII.1997. 